\title{
3-D Atom Probe Tomography of Resin Embedded Samples
}

James E. Evans*, Ilke Arslan**, David L. Jaeger***, Emmanuelle A. Marquis****, Brian P. Gorman*****, Nigel D. Browning****

* Lawrence Livermore National Lab, 7000 East Avenue, Livermore, CA, 94550

** University of California Davis, 1 Shields Avenue, Davis, CA, 95616

*** University of North Texas, 1155 Union Circle \#305310, Denton, TX 76203

**** University of Oxford, Department of Materials, Parks Road, Oxford, UK, OX1 3PH

$* * * * *$ Colorado School of Mines, 1500 Illinois St. Golden, CO, 80401

Atom Probe Tomography (APT) is capable of providing three-dimensional reconstructions of samples with atomic level structural information in addition to the direct elemental composition [1]. When coupled with high-resolution TEM and STEM tomography, APT becomes a very powerful tool for understanding the ultrastructural detail of nanomaterials [2]. However, to date, APT has focused mainly on material science samples such as semiconductors [1]. We sought to utilize laser-assisted APT for the analysis of epoxy resin embedded specimens. The main focus of this research was to determine the feasibility of performing 3-D Atom Probe analysis of biological samples preserved inside a polymer matrix. However, APT of resin embedded samples would also permit the imaging of nanoparticles and other disperse materials at the organic/inorganic interface.

For APT samples, a FIB is used to trench out a sample area of interest, attach small $2 \times 2 \times 5$ micron posts to a coupon and then annular mill these posts to sharpened needle tips required for successful field evaporation [3]. However, for insulating material, there are several issues that arise with the sample preparation process as compared to the standard procedure used for inorganic conductive materials. One such issue relates to the lift-out process using a Focused Ion Beam (FIB). We found that both the epoxy resin and biological protein complexes are extremely sensitive to the FIB and can be affected by the redeposition of removed material, implantation of gallium ions and changes in local structure due to heating or melting of the sample. Thus we have had to reevaluate the protocol used for standard material science sample lift-outs and adjust it accordingly to preserve the integrity of the sample for analysis by minimizing focused ion beam damage. Additionally, since the resin itself is an insulator, it can be difficult to evaporate and once it does, it can have a different evaporation field strength versus the evaporation field of embedded metal particles as is the case for some alloys [4].

In this paper we will discuss the current protocols for sample preparation, results from experiments using a LEAP 3000X and future directions of APT of resin embedded samples for both material science and biological applications. 

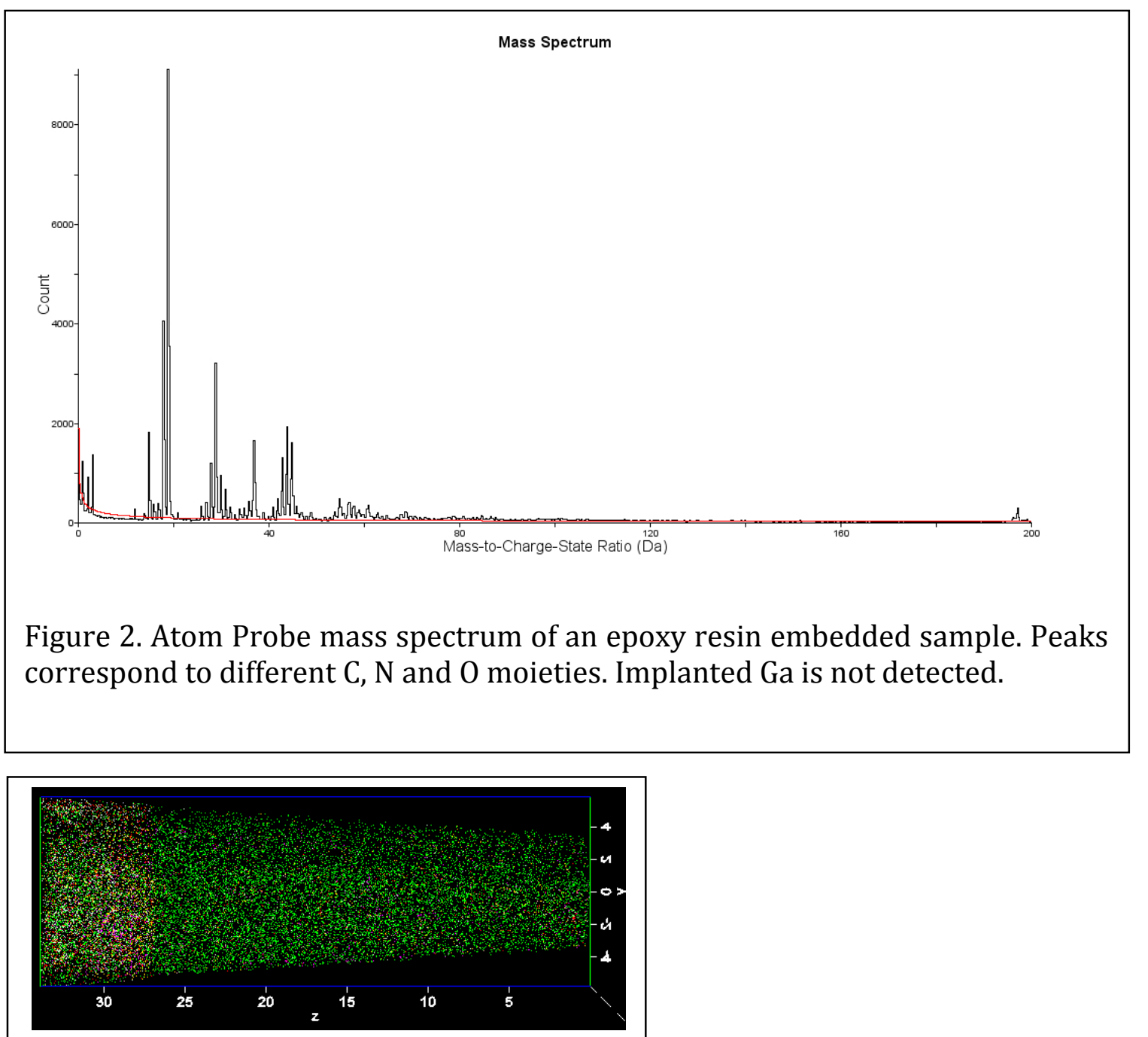

Figure 3. 3-D APT reconstruction showing distribution of carbon moieties of epoxy resin embedded sample.

References:

[1] T.F. Kelly and M.K. Miller, Rev. Sci. Instrum. 78 (2007) 031101

[2] I. Arslan et al., Ultramicroscopy 108 (2008) 1579

[3] M.K. Miller, et al., Micsrosc. Microanal. 13 (2007) 428

[4] E.A. Marquis and F. Vurpillot Micsrosc. Microanal. 14 (2008) 561 